\title{
TWO RESULTS ON FIXED RINGS
}

\author{
J.-L. PASCAUd
}

\begin{abstract}
Let $R$ be a semiprime ring, $G$ a finite group of automorphisms of $R$ and $B$ the algebra of the group. (A) If $R$ is left primitive and $B$ is $G$-simple then the fixed subring $R^{G}$ is left primitive. (B) If $B$ is semiprime and $R^{G}$ is a left Goldie ring, then $R$ can be embedded in a free left $R^{G}$-module of finite rank. Consequently if $R^{G}$ is left Noetherian, $R$ is left Noetherian.
\end{abstract}

Our first result answers a question of J. Fisher and J. Osterburg in [3, Question 11]. The second one generalizes similar results of $D$. Farkas and $R$. Snider when $R$ has no $|G|$-torsion or when $R$ has no nilpotent element [2] and S. Montgomery when $G$ is $X$-outer [7].

Let $R$ be a semiprime ring, $R_{\mathrm{g}}$ the ring of left quotients of $R$ with respect to the filter $\mathcal{F}$ of all nonzero two-sided ideals and $Q$ the left maximal ring of quotients of $R$. Up to isomorphisms, we have $R \subseteq R_{\mathscr{F}} \subseteq Q$. Let $G$ be a finite group of automorphisms of $R$ : the action of $G$ can be extended in a natural way to $R_{\mathscr{F}}$ and we write $s^{g}$ for the image of $s \in R_{\mathscr{G}}$ under $g \in G$. For $g \in G$, let $\Phi_{g}=\{s \in$ $\left.R_{\mathscr{F}} ; \forall r \in R, r s=s r^{8}\right\}$; there exists $x_{g} \in R_{\mathscr{F}}$ such that $\Phi_{g}=C x_{g}[4$, Lemma 6] where $C$ denotes the center of $R_{\mathscr{F}}$ So, on account of the relations $\Phi_{h} \Phi_{g} \subseteq \Phi_{h g}$ and $\Phi_{g}^{h}=\Phi_{h g h^{-1}}$, the "algebra of the group" $B(R ; G)=\Sigma_{g \in G} \Phi_{g}$ is a ring invariant by the action of $G$ (for more details, see [5], [6]). Subsequently we suppose that $C$ is Artinian; so $B$ is semisimple Artinian. $B e_{1}, \ldots, B e_{r}$ (resp. $C \varepsilon_{1}, \ldots, C \varepsilon_{s}$ ) denote the simple components of $B$ (resp. $C$ ). Considering $e \in\left\{e_{1}, \ldots, e_{r}\right\}$ and $\varepsilon \in$ $\left\{\varepsilon_{1}, \ldots, \varepsilon_{s}\right\}$ such that $e \varepsilon=e$ and following [4, Lemma 3], the free product $B e{ }_{C e} C \varepsilon[X]$ contains an element $\mu(X)=\sum_{i=1}^{m} a_{i} X b_{i}$ which commutes with all elements of $B e$ (and even with all elements of $B$ ); we can suppose that $b_{1}, \ldots, b_{m}$ are $C$-independent and $a_{1}, \ldots, a_{m}$ nonzero. $H_{e}=\left\{g \in G ; \varepsilon x_{g} \neq 0\right\}$ is a subgroup of $G$ and, for each $g \in H, \varepsilon^{g}=\varepsilon, \varepsilon x_{g}$ is invertible in $\varepsilon R$ and the action of $g$ on $\varepsilon R$ agrees with that of the inner automorphism defined by $\varepsilon x_{g}[5$, Lemmas 1,2$]$. Let

$$
\tau_{e}(X)=\sum_{k=1}^{n}(\mu(X))^{g_{k}}
$$

where $g_{1}=1, g_{2}, \ldots, g_{n}$ are representatives of the right cosets of $G$ modulo $H_{e} \cdot \tau_{e}$ defines an $R^{G}-R^{G}$-bimodule homomorphism from $R_{\mathcal{F}}$ to $\left(R_{\mathcal{G}}\right)^{G}$ which satisfies:

(a) $\tau_{e}(e x)=\tau_{e}(x)$,

(b) $\forall I \in \mathcal{F}, \tau_{e}(I) \neq 0$,

Received by the editors May 16, 1980 and, in revised form, July 8, 1980.

AMS (MOS) subject classifications (1970). Primary 16A20, 16A72, 16A74.

Key words and phrases. Finite group of automorphisms acting on a ring, primitive ring, semiprime Goldie ring. 
(c) $\exists I_{e} \in \mathscr{F}, \tau_{e}\left(I_{e}\right) \subset R^{G}[5$, pp. 215-216].

(A) In this paragraph, we suppose that $R$ is a left primitive ring, $V$ is a faithful irreducible left $R$-module and that $B$ is $G$-simple (i.e. $B$ has no nontrivial $G$-invariant ideal). Then $C$ is a field and we set $\tau=\tau_{e_{1}}$.

Proposition 1. $V$ is a finitely generated left $R^{G}$-module.

PRoof. $b_{1}$ is left independent of $b_{2}, \ldots, b_{m}, b_{1}^{g_{2}}, \ldots, b_{m}^{g_{2}}, \ldots, b_{1}^{8}, \ldots, b_{m}^{g_{2}}$ relative to the sequence of automorphisms $1, \ldots, 1, g_{2}, \ldots, g_{2}, \ldots, g_{n}, \ldots, g_{n}$ (i.e. $\left.b_{1} \notin \sum_{k=2}^{n} \Phi_{g_{k}} b_{1}^{\beta_{k}}+\sum_{i=2}^{m} \sum_{k=1}^{n} \Phi_{g_{k}} b_{i}^{\beta_{k}}\right)$; so, by [5, Proposition 1'] there exist elements $v_{j}, t_{j} \in R$ such that $\alpha=\Sigma_{j} v_{j} b_{1} t_{j} \neq 0$ and $\Sigma_{j} v_{j}^{8} b_{i}^{8} t_{j}=0$ for all $g \in$ $\left\{g_{2}, \ldots, g_{n}\right\}$ if $i=1$ and all $g \in\left\{g_{1}, \ldots, g_{n}\right\}$ if $i \neq 1$. This implies

$$
a_{1} x \alpha=\sum_{j} a_{1} x v_{j} b_{1} t_{j} \text { and } \sum_{j}\left(a_{i} x v_{j}\right)^{8} b_{i}^{8} t_{j}=0
$$

for all $x \in R$, all $g \in\left\{g_{2}, \ldots, g_{n}\right\}$ if $i=1$ and all $g \in\left\{g_{1}, \ldots, g_{n}\right\}$ if $i \neq 1$. Summing these relations we obtain

$$
\forall x \in R \quad a_{1} x \alpha=\sum_{j} \tau\left(x v_{j}\right) t_{j} .
$$

Now, by definition of $R_{\mathscr{F}}$ and by (c) there exists a $G$-invariant ideal $J \in \mathscr{F}$ such that $J \alpha \subseteq R$ and that $\tau\left(J v_{j}\right) \subseteq R^{G}$ for each $j$. Consider the set $T$ of elements $\alpha \in R$ such that there exist a $G$-invariant ideal $I \in \mathscr{F}$, mappings $f_{j}: I \rightarrow R^{G}$ and elements $t_{j} \in R$ which satisfy

$$
\forall x \in I \quad a_{1} x \alpha=\sum_{j} f_{j}(x) t_{j} .
$$

From the foregoing remarks, $T$ is a nonzero two-sided ideal of $R$. Thus, since $V$ is a faithful irreducible left $R$-module, for $0 \neq w \in V$ we have $V=R w=T w$. Therefore we can consider $\alpha_{0} \in T$ with $w=\alpha_{0} w$ so that

$$
\forall x \in I \quad a_{1} x w=a_{1} x \alpha_{0} w=\sum_{j} f_{j}(x) t_{j} w .
$$

Since $V=I w$, it follows that $a_{1} V \subseteq \Sigma_{j} R^{G_{t} w}$. By letting $h \in G$ act on relation (1) and applying the previous method we obtain also $a_{1}^{h} V \subseteq \Sigma_{j} R^{G} t_{j}^{h} w$. Let $W=$ $\Sigma_{g \in G} \Sigma_{j} R^{G} t_{j}^{g} w$; then the set $A$ of elements $a \in R$ which satisfy

$$
\forall h \in G \quad a^{h} V \subseteq W
$$

is a nonzero $G$-invariant sub- $R^{G}-R$-bimodule of $R_{\mathscr{F}}$ Its left annihilator in $B$ is a $G$-invariant ideal; hence this annihilator is zero. But, by [5, Lemma 5], there exists $K \in \mathscr{F}$ such that $K \subseteq A$; this implies $V=K V \subseteq W$.

Proposition 2. $R^{G}$ is left primitive.

Proof. By Proposition 1, $V$ contains a maximal left $R^{G}$-module $W$. Let $\mathscr{T}=\{a$ $\left.\in R ; \forall g \in G, a^{g} V \subseteq W\right\}$; this is a sub- $R^{G}-R$-bimodule of $R_{\mathscr{G}}$ which is $G$ invariant. Suppose that $\mathcal{T}$ is nonzero. Its left annihilator in $B$ (which is a $G$ invariant ideal of $B$ ) is zero and [5, Lemma 5] there exists $K \in \mathscr{F}$ such that 
$K \subseteq \mathcal{T}$; hence $V=K V \subseteq W$, a contradiction. Thus $\mathcal{T}$ is zero and $V / W$ is a faithful irreducible left $R^{G}$-module.

(B) In this part, we suppose that $B$ is semiprime and $R^{G}$ is a left Goldie ring. V. K. Kharchenko has proved that $R$ is a left Goldie ring; the action of $G$ can be extended to $Q$ and $Q^{G}$ is the left maximal ring of quotients of $R^{G}$ [5, Theorem 9]. $C$ is the center of $Q$ and $B(R ; G)=B(Q ; G)[1$, Proposition 1$]$; since $Q$ is semisimple Artinian, $C$ is Artinian. Theorem 10 of [5] shows that $Q$ is a finitely generated right $Q^{G}$-module; there exist elements $x_{1}, \ldots, x_{t}$ of $R$ such that $Q=$ $\sum_{k=1}^{t} x_{k} Q^{G}$.

Proposition 3. $R$ can be embedded in a free left $R^{G}$-module of finite rank.

Proof. Let $I=\bigcap_{j=1}^{r} I_{e_{j}} \in \mathcal{F}$ and

$$
f: I \rightarrow\left(R^{G}\right)^{r t|G|}, \quad a \rightarrow\left(\tau_{e_{j}}\left(a^{g} x_{k}\right)\right), \quad 1<j<r, 1<k<t, g \in G .
$$

$f$ defines a left $R^{G}$-module homomorphism. Since $R$ is a semiprime Goldie ring, $I$ contains a regular element so that $R$ can be embedded in $I$; hence it is sufficient to prove that $f$ is injective.

Let $a$ be a nonzero element of $I$. Then $A=\Sigma_{g \in G} a^{g} R$ is a nonzero $G$-invariant right ideal. Its left annihilator $l_{B}(A)$ in $B$ is $G$-invariant; therefore $l_{B}(A)$ is a two-sided ideal generated by a central idempotent $e \neq 1$ of $B$ and there exists $J \in \mathcal{F}$ such that $(1-e) J \subseteq R^{G} A\left[5\right.$, Lemma 5]. We choose $e_{j}$ such that $e_{j}(1-e)$ $=e_{j}$. From $\tau_{e_{j}}(x)=\tau_{e_{j}}\left(e_{j} x\right)$, we obtain the following relations:

$$
\tau_{e_{j}}\left(R^{G} A\right) \supseteq \tau_{e_{j}}((1-e) J)=\tau_{e_{j}}\left(e_{j}(1-e) J\right)=\tau_{e_{j}}\left(e_{j} J\right)=\tau_{e_{j}}(J) .
$$

Thus $\tau_{e_{j}}(J)$ and $\tau_{e}(A)$ are nonzero. Moreover, since $B$ is contained in the centralizer of $Q^{G}$ in $Q$, we have that $\tau_{e_{j}}$ defines a $Q^{G}$-right module homomorphism from $Q$ to $Q^{G}$. Then, from $\tau_{e_{j}}(A Q)=\sum_{g \in G} \Sigma_{k=1}^{t} \tau_{e}\left(a^{g} x_{k}\right) Q^{G}$ being nonzero, there exist some $g \in G$ and some $k$ such that $\tau_{e_{j}}\left(a^{g} x_{k}\right)$ is nonzero.

REMARK. A (not necessarily finite) group of automorphisms of a semiprime ring $R$ is an " $M$-group" if the algebra $B$ of $G$ is semiprime and finitely generated as a $C$-module and if, in the set $E$ of nonzero central idempotents of $R$, the set of idempotents $e$ such that $H_{e}=\left\{g \in G ; \forall e_{1} \in E, e_{1}<e, e_{1} \Phi_{g} \neq 0\right\}$ is a subgroup of finite index in $G$, forms a cofinal system. In our hypothesis ( $B$ is semiprime and $C$ is Artinian) it means that for each $j(1<j<s) H_{g}$ is of finite index. All Kharchenko's results used in our proofs are available for $M$-groups; so, the foregoing results are more generally true for $M$-groups.

\section{REFERENCES}

1. M. Cohen and S. Montgomery, The normal closure of a semi-prime ring, (Proc. 1978 Antwerp Conf.), Ring Theory, Dekker, New York, 1979, pp. 43-59.

2. D. Farkas and R. Snider, Noetherian fixed rings, Pacific J. Math. 69 (1977), 347-353. 
3. J. Fisher and J. Osterburg, Finite actions on non commutative rings: a survey since 1970, (Proc. 3rd Oklahoma Conf., 1979), Ring Theory and Algebra III, Dekker, New York, 1980.

4. V. K. Kharchenko, Fixed elements under a finite group acting on a semi-prime ring, Algebra and Logic 14 (1976), 203-213.

5. __ Galois theory of semi-prime rings, Algebra and Logic 16 (1978), 208-258.

6. S. Montgomery, Fixed rings of finite automorphism groups of associative rings, Lecture Notes in Math., vol. 818, Springer-Verlag, Berlin and New York.

7. , Outer automorphisms of semi-prime rings, J. London Math. Soc. 18 (1978), 209-221.

Département de Mathématiques, Université de Poitiers, 40 avenue du Recteur Pineau, 86022 Portiers, France 